\title{
Fatores Clínico-Laboratoriais Associados ao Internamento Prolongado em Doentes com Celulite/Erisipela
}

\section{Clinical and Laboratory Factors Associated with Prolonged Hospital Stay among Patients with Cellulitis/Erysipelas}

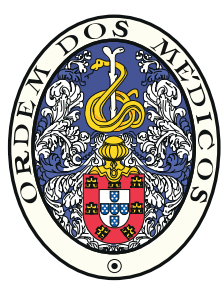

\author{
Ângela RODA® ${ }^{1}$, Ana Marcos PINTO ${ }^{1}$, Ana Rita FILIPE², Ana Rita TRAVASSOS ${ }^{1}$, João Pedro FREITAS ${ }^{1}$, \\ Paulo FILIPE $1,3,4$ \\ Acta Med Port 2019 Jun;32(6):448-452 - https://doi.org/10.20344/amp.10735
}

\section{RESUMO}

Introdução: A celulite e a erisipela constituem a causa mais frequente de internamento no Serviço de Dermatologia do Hospital Santa Maria. Este estudo teve como objetivo investigar se as características demográficas, as comorbilidades, a existência de episódios prévios de celulite/erisipela, a presença de complicações associadas, os parâmetros laboratoriais na admissão, o isolamento de microrganismo em cultura ou o uso prévio de antibióticos estão associados a internamentos prolongados.

Material e Métodos: Estudo retrospetivo, incluindo os doentes internados no Serviço de Dermatologia do Hospital Santa Maria com o diagnóstico de celulite/erisipela, entre 1 de julho de 2012 e 30 de junho de 2017.

Resultados: Existiram 372 internamentos, correspondendo a 348 doentes. A mediana do tempo de internamento foi de 11 dias. A idade $(p=0,002$, OR 1,03, 95\% IC 1,01-1,04), a existência de internamento prévio por celulite/erisipela $(p=0,005$, OR 4,81, $95 \%$ IC 1,63 - 14,23), a presença de complicações associadas à celulite/erisipela $(p=0,001$, OR 3,28, 95\% IC 1,63-6,59), a leucocitose $(p=0,049$, OR $1,81,95 \%$ IC $1,00-3,30)$, valores elevados de proteína C reativa $(p=0,035$, OR 1,03, 95\% IC 1,00 - 1,06) e o isolamento de microrganismo em cultura $(p=0,002$, OR 2,59, 95\% IC 1,41-4,79) estiveram associados a internamentos prolongados. Discussão: A par dos maiores custos associados, o internamento prolongado por celulite/erisipela está frequentemente associado à necessidade de investigação clínica adicional, a tratamentos invasivos, a cursos prolongados de antibioterapia, ao risco de infeções nosocomiais e ao atraso no retorno às atividades da vida diária. Assim, o estudo dos fatores clínico-laboratoriais associados ao internamento prolongado por celulite/erisipela é fundamental e poderá ser útil para a construção de um score de gravidade.

Conclusão: O conhecimento de características clínicas e laboratoriais associadas ao internamento prolongado poderá ser relevante para melhorar os cuidados de saúde, através da redução dos tempos de internamento e dos seus riscos e custos associados.

Palavras-chave: Celulite; Erisipela; Hospitalização; Leucocitose; Proteína C-Reactiva

\section{ABSTRACT}

Introduction: Cellulitis and erysipelas represent the most frequent cause of hospitalization in the dermatology department of Santa Maria Hospital in Lisbon, Portugal. The aim of this study was to investigate whether patient demographics, comorbidities, previous episodes of cellulitis/erysipelas, the presence of complications, laboratory markers at admission, microbial isolation or previous use of antibiotics, are associated with prolonged stays.

Material and Methods: Retrospective analysis, including patients admitted with cellulitis/erysipelas in the inpatient dermatology department of Santa Maria Hospital between July $1^{\text {st }} 2012$ and June 30 2017.

Results: There were 372 admissions, corresponding to 348 patients. The median length of stay was 11 days. Increased age $(p=0.002$, OR $1.03,95 \% \mathrm{Cl} 1.01-1.04)$, previous episode of cellulitis/erysipelas requiring hospitalization $(p=0.005, \mathrm{OR} 4.81$, $95 \% \mathrm{Cl} 1.63-14.23)$, the presence of cellulitis/erysipelas-associated complications ( $p=0.001, \mathrm{OR} 3.28,95 \% \mathrm{Cl} 1.63-6.59)$, leukocytosis $(p=0.049$, OR $1.81,95 \% \mathrm{Cl} 1.00-3.30)$, high levels of C-reactive protein $(p=0.035$, OR $1.03,95 \% \mathrm{Cl} 1.00-1.06)$ and a positive culture result ( $p=0.002$, OR $2.59,95 \% \mathrm{Cl} 1.41-4.79$ ) were associated with prolonged hospitalization.

Discussion: Prolonged hospitalization for cellulitis/erysipelas is associated with higher costs, additional clinical investigation, invasive treatments, prolonged courses of antibiotic therapy, risk of nosocomial infections, and delayed return to activities of daily living. Thus, the investigation of clinical-laboratory factors associated with prolonged hospitalization for cellulitis / erysipelas is essential and may be useful for the construction of a severity score.

Conclusion: The knowledge of the characteristics that are associated with prolonged stay among patients with cellulitis/erysipelas may be relevant to improve health care, by reducing the length of hospital stay and associated risks and costs.

Keywords: C-Reactive Protein; Cellulitis; Erysipelas; Hospitalization; Leukocytosis

\section{INTRODUÇÃO}

A celulite e a erisipela constituem a causa mais frequente de internamento no Serviço de Dermatologia do Hospital Santa Maria, representando cerca de $18 \%$ das admissões no serviço anualmente.

Classicamente, existe uma distinção semiológica entre celulite e erisipela. Contudo, esta diferenciação baseia-se em critérios clínicos pouco precisos e carece de relevância na abordagem e tratamento da patologia em si. Deste modo, é frequente o uso indiscriminado de ambos os termos, na prática clínica. ${ }^{1}$

Até à data, o conhecimento dos fatores que influenciam a duração de internamento dos doentes com celulite/erisipela é escasso. O objetivo deste estudo foi investigar se as

4. Instituto de Medicina Molecular. Faculdade de Medicina. Universidade de Lisboa. Lisboa. Portugal.

$\triangle$ Autor correspondente: Ângela Roda. angela.neto.roda@gmail.com

Recebido: 28 de abril de 2018 - Aceite: 02 de outubro de 2018| Copyright @ Ordem dos Médicos 2019 
características demográficas, as comorbilidades, a existência de episódios prévios de celulite/erisipela, a presença de complicações associadas à celulite/erisipela, os parâmetros laboratoriais de infeção na admissão, o isolamento de microrganismos em cultura ou o uso prévio de antibiótico, estão associados a um internamento prolongado.

\section{MATERIAL E MÉTODOS}

Desenvolveu-se um estudo retrospetivo, no qual se incluíram os doentes com idade superior ou igual a 18 anos internados no Serviço de Dermatologia do Hospital de Santa Maria com o diagnóstico de celulite ou erisipela, independentemente da localização, entre 1 de julho de 2012 e 30 de junho de 2017.

Foram avaliadas as seguintes variáveis explicativas: características demográficas, como idade e sexo; a existência de comorbilidades, nomeadamente diabetes mellitus, doença vascular periférica (incluindo venosa, arterial e linfática), imunodepressão (incluindo a de etiologia infeciosa, hematológica, farmacológica) e cirurgia local prévia; antecedentes de celulite/erisipela, nomeadamente a existência de pelo menos um episódio prévio de celulite/erisipela, a existência de pelo menos um internamento prévio por celulite/ erisipela e o cumprimento de profilaxia antibiótica; a existência de complicações associadas à celulite/ erisipela (incluindo abcesso, linfangite, necrose, ulceração, bolha, envolvimento osteoarticular); a existência de porta de entrada (incluindo todas as condições que causem disrupção da barreira cutânea, como infeções fúngicas (onicomicose, tinha), virais (infeções herpéticas), bacterianas (foliculites, furúnculos), feridas traumáticas, feridas cirúrgicas, picadas de inseto); parâmetros laboratoriais na admissão, nomeadamente contagem do número de leucócitos, contagem do número de neutrófilos, valor de proteína $\mathrm{C}$ reativa $(\mathrm{PCR})$; isolamento de microrganismo em cultura (através de exame bacteriológico do exsudado existente em ulcerações ou abcessos associados e/ou exame bacteriológico do sangue); uso de antibiótico na semana anterior à admissão para o tratamento do episódio atual de celulite/erisipela.

A seleção das variáveis teve em consideração o conhecimento da fisiopatologia da doença e as suas relações hipotéticas com a duração do internamento.

\section{Análise estatística}

Na análise descritiva, as variáveis categóricas são apresentadas como frequências e percentagens e as variáveis contínuas como médias e desvio-padrão, ou medianas e amplitude interquartílica, consoante a sua distribuição seja normal ou não, respetivamente. Para verificar a normal distribuição dos dados, recorreu-se ao teste de Shapiro-Wilk ou à análise dos valores de assimetria e achatamento baseada no ponto de corte $[-1 ; 1]$.

Os internamentos foram divididos em dois grupos distintos, consoante a sua duração fosse superior a 14 dias ou inferior ou igual a 14 dias. Esta dicotomização foi definida a posteriori, com base na mediana da duração de internamento da nossa amostra. Os internamentos com duração superior a 14 dias foram considerados prolongados.

Relativamente à análise bivariada, utilizou-se o teste do qui-quadrado ou o teste exato de Fisher para averiguar as associações entre as variáveis categóricas e o teste $t$ para avaliação das variáveis contínuas.

Por último, elaborou-se um modelo de regressão logística otimizado, com método de entrada de dados stepwise, incluindo as variáveis estatisticamente significativas na

Tabela 1 - Análise univariada das características dos doentes admitidos por celulite/erisipela

\begin{tabular}{|c|c|c|c|c|}
\hline & $\begin{array}{c}\text { Geral } \\
(n=372)\end{array}$ & $\begin{array}{c}\text { Internamento } \\
\text { curto } \\
(n=247)\end{array}$ & $\begin{array}{c}\text { Internamento } \\
\text { prolongado } \\
(n=125)\end{array}$ & Valor $p$ \\
\hline Idade - média (DP) (anos) & $61,2(18,4)$ & $59,5(18,7)$ & $64,5(17,4)$ & 0,01 \\
\hline Sexo feminino $-\mathrm{n}(\%)$ & $191(51 \%)$ & $122(49 \%)$ & $69(55 \%)$ & 0,29 \\
\hline Diabetes mellitus - n (\%) & $86(23 \%)$ & $50(20 \%)$ & $36(29 \%)$ & 0,06 \\
\hline Doença vascular - n (\%) & $96(26 \%)$ & $55(22 \%)$ & $41(33 \%)$ & 0,03 \\
\hline Imunodepressão - n (\%) & $47(13 \%)$ & $29(12 \%)$ & $18(14 \%)$ & 0,47 \\
\hline Cirurgia local prévia - n (\%) & $83(22 \%)$ & $51(21 \%)$ & $32(26 \%)$ & 0,28 \\
\hline Episódio(s) prévio(s) de celulite/erisipela - n (\%) & $79(21 \%)$ & $50(20 \%)$ & $29(23 \%)$ & 0,51 \\
\hline Internamento(s) prévio(s) por celulite/erisipela - n (\%) & $24(6 \%)$ & $8(3 \%)$ & $16(13 \%)$ & $<0,001$ \\
\hline Uso de antibioterapia profilática & $15(4 \%)$ & $9(4 \%)$ & $6(5 \%)$ & 0,59 \\
\hline Complicações - n (\%) & $66(18 \%)$ & $32(13 \%)$ & $34(27 \%)$ & 0,001 \\
\hline Porta de entrada - n (\%) & $193(52 \%)$ & $129(52 \%)$ & $64(51 \%)$ & 0,85 \\
\hline Leucocitose - n (\%) & $188(51 \%)$ & $109(44 \%)$ & $79(63 \%)$ & 0,001 \\
\hline Neutrofilia - n (\%) & $221(59 \%)$ & $135(55 \%)$ & $86(69 \%)$ & 0,009 \\
\hline PCR - media (DP) (mg/dL) & $12,4(9,9)$ & $11,2(8,9)$ & $14,8(11,5)$ & 0,002 \\
\hline Isolamento de microrganismo em cultura* - n (\%) & $82(22 \%)$ & $37(15 \%)$ & $45(36 \%)$ & $<0,001$ \\
\hline Uso de antibiótico na semana anterior - $\mathrm{n}(\%)$ & $111(30 \%)$ & $67(27 \%)$ & $44(35 \%)$ & 0,11 \\
\hline
\end{tabular}


Tabela 2 - Complicações associadas à celulite/erisipela

\begin{tabular}{lccc}
\hline & $\begin{array}{c}\text { Geral } \\
(\mathbf{n = 6 6 )}\end{array}$ & $\begin{array}{c}\text { Internamento curto } \\
(\mathbf{n = 3 2 )}\end{array}$ & $\begin{array}{c}\text { Internamento prolongado } \\
(\mathbf{n = 3 4 )}\end{array}$ \\
\hline Abcesso $-\mathbf{n}(\%)$ & $33(50 \%)$ & $11(35 \%)$ & $22(64 \%)$ \\
Linfangite $-\mathbf{n}(\%)$ & $22(33 \%)$ & $18(56 \%)$ & $4(12 \%)$ \\
Ulceração $-\mathbf{n}(\%)$ & $4(6 \%)$ & $1(3 \%)$ & $3(9 \%)$ \\
Necrose $-\mathbf{n}(\%)$ & $1(2 \%)$ & $0(0 \%)$ & $1(3 \%)$ \\
Bolha - $\mathbf{n}(\%)$ & $2(3 \%)$ & $1(3 \%)$ & $1(3 \%)$ \\
Envolvimento osteoarticular $-\mathbf{n}(\%)$ & $4(6 \%)$ & $1(3 \%)$ & $3(9 \%)$ \\
\hline
\end{tabular}

análise bivariada, com o intuito de determinar quais as variáveis associadas ao internamento prolongado.

Todos os valores $p$ reportados são bicaudados, com um nível de significância ( $\alpha$ ) de 0,05. A análise de dados foi realizada com recurso ao software SPSS, versão 23.

\section{RESULTADOS}

Durante os cinco anos analisados, existiram 372 internamentos, correspondendo a 348 doentes. A mediana da duração de internamento foi de 11 dias. Em relação à localização da celulite/erisipela, o membro inferior foi o local mais frequentemente envolvido ( $n=284,76 \%)$, seguido da face $(n=42,11 \%)$ e do membro superior ( $n=36,10 \%)$. Quarenta e quatro por cento dos internamentos foram prolongados $(\mathrm{n}=125)$.

As características dos doentes admitidos por celulite/ erisipela estão referidas na Tabela 1. Como se pode constatar, a média de idades foi significativamente superior no grupo do internamento prolongado $[64,5(17,4)$ vs 59,5 $(18,7) ; p=0,01]$. Em relação ao sexo, não houve uma diferença estatisticamente significativa entre ambos os grupos
( $p=0,29$ ), embora a maioria dos doentes com internamento prolongado fosse do sexo feminino. As frequências de diabetes mellitus, doença vascular, imunodepressão e cirurgia local prévia foram de $29 \%$ vs $20 \%$, $33 \%$ vs $22 \%$, $14 \%$ vs $12 \%$ e $26 \%$ vs $21 \%$, respetivamente para o internamento prolongado e curto. Em $21 \%$ dos internamentos, havia história prévia de celulite/erisipela e, inclusivamente, em $6 \%$ dos casos, já tinha existido pelo menos um internamento anterior pelo mesmo motivo. Em relação ao episódio de celulite/erisipela que motivou o internamento, a existência de complicações foi significativamente superior entre os doentes com internamento prolongado $(27 \%$ vs $13 \% ; p=0,001)$. A discriminação das complicações associadas à celulite/erisipela encontra-se na Tabela 2. A identificação de porta de entrada foi semelhante em ambos os grupos (52\% vs 51\%). Foram realizadas culturas em 262 internamentos, com isolamento de microrganismo em $31 \%$ ( $n=82$ ) dos casos (72 casos com isolamento de microrganismo em exame bacteriológico de exsudado, sete casos em hemocultura e três casos em exame bacteriológico do exsudado e hemocultura). Na Fig. 1 especificam-se os

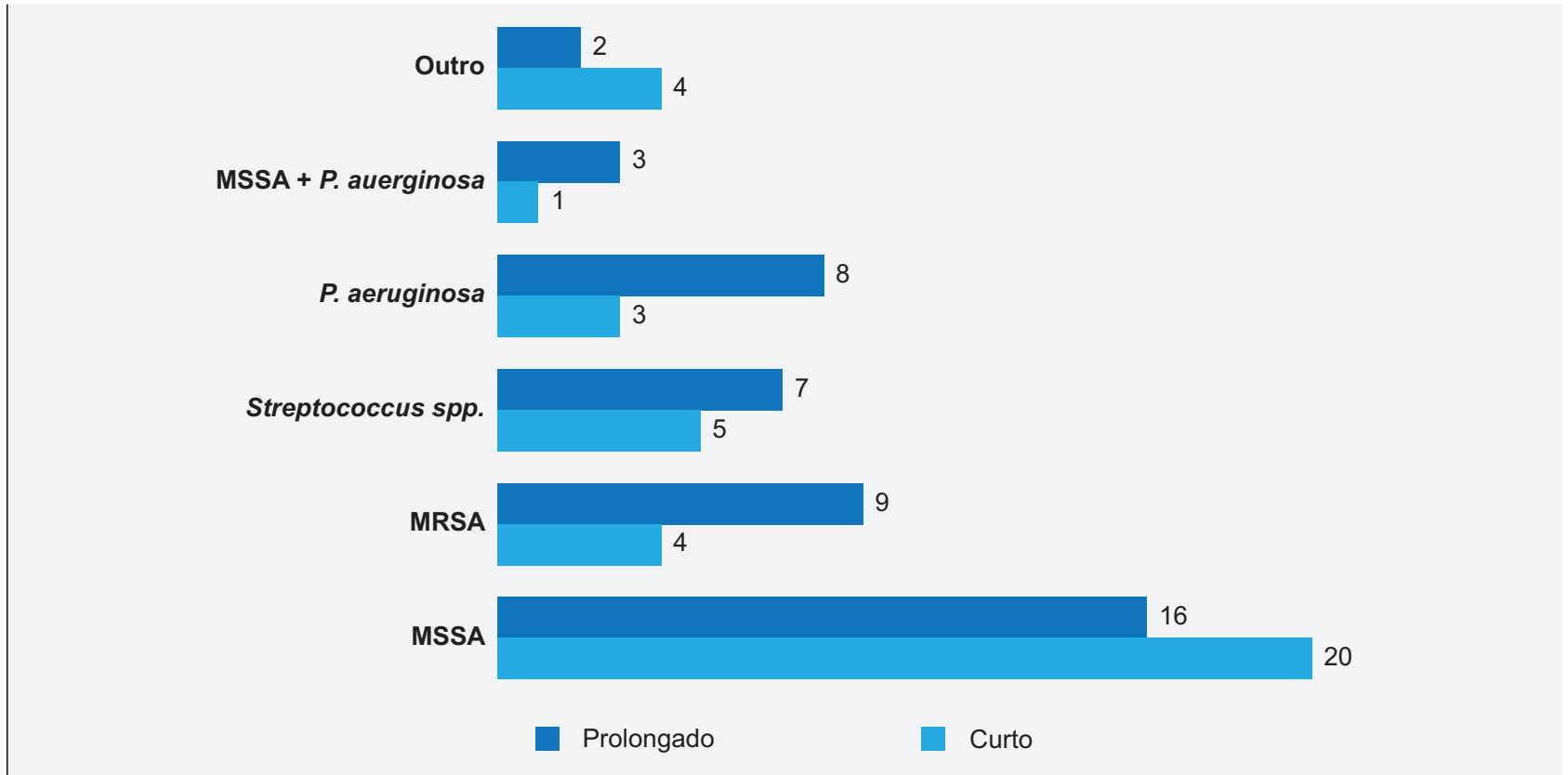

Figura 1 - Microrganismos isolados em cultura.

MSSA: Staphylococcus aureus meticilino-sensível; MRSA: Staphylococcus aureus meticilino-resistente; P. aeruginosa: Pseudomonas aeruginosa; spp.: espécie. Outro, inclui: Serratia marcescens $(n=3)$, E. coli $(n=1)$, Klebsiella pneumoniae $(n=1)$, Pantoea agglomerans $(n=1)$. 
Tabela 3 - Regressão logística otimizada, incluindo 70\% dos dados (262/372) devido aos missing values

\begin{tabular}{lccc}
\hline & Valor $\boldsymbol{p}$ & Odds ratio ajustadas & Intervalo de confiança 95\% \\
\hline Idade & 0,002 & 1,03 & $1,01-1,04$ \\
Internamento(s) prévio(s) por celulite/erisipela & 0,005 & 4,81 & $1,63-14,23$ \\
Doença vascular & 0,42 & 1,30 & $0,69-2,46$ \\
Complicações & 0,001 & 3,28 & $2,63-6,59$ \\
Leucocitose & 0,049 & 1,81 & $1,00-3,30$ \\
PCR & 0,035 & 1,03 & $1,00-1,06$ \\
Isolamento de microrganismo em cultura* & 0,002 & 2,59 & $1,41-4,79$ \\
\hline
\end{tabular}

microrganismos isolados. Por último, a utilização de antibiótico na semana anterior à admissão, embora tenha sido superior no grupo de internamento prolongado (35\% vs $27 \%$ ), a diferença entre os grupos não foi estatisticamente significativa $(p=0,11)$.

O ajustamento do modelo de regressão logística permitiu identificar como fatores associados ao internamento prolongado a idade $(p=0,002)$, o internamento prévio por celulite/erisipela $(p=0,005)$, a presença de complicações $(p=0,001)$, a leucocitose $(p=0,049)$, o valor de PCR ( $p=0,035)$ e o isolamento de microrganismo em cultura $(p=0,002)$ (Tabela 3$)$. As variáveis com maior preponderância para o internamento prolongado foram a existência de internamentos prévios por celulite/erisipela e a presença de complicações associadas.

No que diz respeito à publicação de informação pessoal, os autores declaram ter seguido os protocolos em vigor na Instituição de Saúde onde se encontram afiliados. A informação foi anonimizada e nenhum dos autores teve acesso a elementos identificativos dos doentes. $O$ estudo foi realizado de acordo com a Declaração de Helsínquia da Associação Médica Mundial.

\section{DISCUSSÃO}

Este é o primeiro estudo realizado em Portugal que investiga os fatores clínico-laboratoriais associados ao internamento prolongado de doentes admitidos com o diagnóstico de celulite/erisipela. Assim, observámos que a idade, a existência de pelo menos um internamento prévio por celulite/erisipela, a presença de complicações, a leucocitose e o valor de PCR no momento da admissão e o isolamento de microrganismo em cultura estiveram associados ao internamento prolongado.

A par dos maiores custos associados, o internamento prolongado por celulite/erisipela, está frequentemente associado à necessidade de investigação clínica adicional com exames complementares de diagnóstico como (por exemplo, exames imagiológicos para a exclusão de eventuais complicações associadas), a tratamentos invasivos, como drenagens ou desbridamentos cirúrgicos, a cursos prolongados de antibioterapia, com o risco inerente de desenvolvimento de resistências aos antimicrobianos, ao risco de infeções nosocomiais e ao atraso no retorno às atividades da vida diária, com impacto na qualidade de vida do doente.

Embora a terapêutica antibiótica ideal e respetiva duração ainda não estejam estabelecidas, o nosso serviço segue um protocolo terapêutico que se baseia na presença ou ausência de comorbilidades e na toma prévia de antibiótico. Apesar de terem sido propostas normas de orientação clínica para a abordagem e tratamento de doentes com celulite/erisipela, ${ }^{2,3}$ ainda existem poucos dados que permitam identificar os doentes em risco de internamento prolongado e que, por sua vez, beneficiem de uma terapêutica mais agressiva.

À semelhança do nosso estudo, outros estudos investigaram as características associadas ao internamento prolongado de doentes com celulite/erisipela. De acordo com o estudo multicêntrico realizado por Gang et al nos Estados Unidos da América, onde foram incluídos 4224 internamentos por celulite do membro inferior, concluiu-se que variáveis como a idade, o sexo feminino, a diabetes mellitus, a taquicardia, a hipotensão, a leucocitose, a neutrofilia e a elevação da creatinina sérica estiveram associadas ao internamento prolongado. ${ }^{4}$ Num estudo da Nova Zelândia, onde foram avaliados 51 doentes admitidos por celulite do membro inferior, mostrou-se que a contagem elevada de neutrófilos, o score de edema e o uso de diuréticos estiveram independentemente associados ao internamento prolongado. ${ }^{5}$ Em 2003, Carratalà et al observaram 332 doentes hospitalizados por celulite, independentemente da sua localização, e concluíram que os doentes com múltiplas comorbilidades, hipoalbuminémia, insuficiência renal ou necrose cutânea à admissão tinham internamentos mais prolongados. ${ }^{6}$ De igual modo, um estudo australiano investigou 395 episódios de celulite/erisipela de diversos locais anatómicos e demonstrou que a idade superior a 60 anos, a duração da sintomatologia superior a 4 dias, a presença de hipoalbuminémia, a existência de bacteriemia e o isolamento de Staphylococcus aureus meticilino-resistente (MRSA) estiveram associados ao internamento prolongado. Por outro lado, a neutrofilia e a elevação da creatinina não estiveram associados ao internamento prolongado nessa análise. ${ }^{7}$ Por último, um estudo canadiano contemplando a sua base de dados nacional com 65454 doentes admitidos por celulite, mostrou que os factores associados ao internamento prolongado foram a idade superior a 65 anos, o sexo feminino e a insuficiência cardíaca congestiva. ${ }^{8}$

Assim, os resultados dos diversos estudos são ainda heterogéneos, podendo estar influenciados pela ausência de critérios definidos para o diagnóstico e abordagem de celulite/erisipela, o que inevitavelmente se traduz numa grande variabilidade de atitudes terapêuticas adotadas nos vários centros, assim como na duração média de interna- 
mento. $^{9-11}$ No entanto, Gang et al defendem que os resultados destes estudos poderão constituir uma ferramenta importante para a construção de um score de gravidade ${ }^{4}$ à semelhança dos scores aplicados nos casos de pneumonia, que levaram à redução das taxas de admissão hospitalar, assim como do tempo de internamento e complicações associadas. ${ }^{12-15}$

Uma das principais limitações deste estudo observacional é a eventual exclusão de potenciais fatores de confundimento para internamento prolongado, como o contexto social adverso ou ocorrência de outras intercorrências não relacionadas diretamente com o episódio de celulite/ erisipela. Contudo, a aplicação do modelo de regressão logística otimizado, com método de entrada de dados stepwise pretende mitigar o impacto estatístico dos fatores de confundimento.

\section{CONCLUSÃO}

Em conclusão, o conhecimento de características clínicas e laboratoriais preditivos de internamento prolongado poderá ser relevante para melhorar os cuidados de saúde, com o intuito de reduzir os tempos de internamento e os seus riscos e custos associados.

\section{REFERÊNCIAS}

1. Caetano M, Amorim I. Erysipelas. Acta Med Port. 2005;18:385-93.

2. Stevens DL, Bisno AL, Chambers HF, Dellinger EP, Goldstein EJ, Gorbach SL, et al. Practice guidelines for the diagnosis and management of skin and soft tissue infections: 2014 update by the Infectious Diseases Society of America. Clin Infect Dis. 2014;59:e10-52.

3. Eron LJ, Lipsky BA, Low DE, Nathwani D, Tice AD, Volturo GA. Expert panel on managing skin and soft tissue infections. Managing skin and soft tissue infections: expert panel recommendations on key decision points. J Antimicrob Chemother. 2003;52:13-17.

4. Garg A, Lavian J, Lin G, Sison C, Oppenheim M, Koo B. Clinical characteristics associated with days to discharge among patients admitted with a primary diagnosis of lower limb cellulitis. J Am Acad Dermatol. 2017;76:626-31.

5. Morpeth SC, Chambers ST, Gallagher K, Frampton C, Pithie AD. Lower limb cellulitis: features associated with length of hospital stay. J Infect. 2006;52:23-9.

6. Carratalà J, Rosón B, Fernández-Sabé N, Shaw E, del Rio O, Rivera $A$, et al. Factors associated with complications and mortality in adult patients hospitalized for infectious cellulitis. Eur J Clin Microbiol Infect Dis. 2003;22:151-7.

7. Figtree M, Konecny P, Jennings Z, Goh C, Krilis SA, Miyakis S. Risk stratification and outcome of cellulitis admitted to hospital. J Infect. 2010;60:431-9.

8. Baibergenova A, Drucker AM, Shear NH. Hospitalizations for cellulitis in Canada: a database study. J Cutan Med Surg. 2014;18:33-7.

\section{PROTECÇÃO DE PESSOAS E ANIMAIS}

Os autores declaram que os procedimentos seguidos estavam de acordo com os regulamentos estabelecidos pelos responsáveis da Comissão de Investigação Clínica e Ética e de acordo com a Declaração de Helsínquia da Associação Médica Mundial.

\section{CONFIDENCIALIDADE DOS DADOS}

Os autores declaram ter seguido os protocolos do seu centro de trabalho acerca da publicação de dados.

\section{CONFLITOS DE INTERESSE}

Os autores declaram não ter conflitos de interesses relacionados com o presente trabalho.

\section{FONTES DE FINANCIAMENTO}

Este trabalho não recebeu qualquer tipo de suporte financeiro de nenhuma entidade no domínio público ou privado.

9. Levell NJ, Wingfield CG, Garioch JJ. Severe lower limb cellulitis is best diagnosed by dermatologists and managed with shared care between primary and secondary care. Br J Dermatol. 2011;164:1326-8.

10. Arakaki RY, Strazzula L, Woo E, Kroshinsky D. The impact of dermatology consultation on diagnostic accuracy and anti- biotic use among patients with suspected cellulitis seen at outpatient internal medicine offices: a randomized clinical trial. JAMA Dermatol. 2014;150:1056-61.

11. Strazzula L, Cotliar J, Fox LP, Hughey L, Shinkai K, Gee SN, et al. Inpatient dermatology consultation aids diagnosis of cellulitis among hospitalized patients: a multi-institutional analysis. J Am Acad Dermatol. 2015;73:70-5.

12. Dean NC, Silver MP, Bateman KA, James B, Hadlock CJ, Hale D. Decreased mortality after implementation of a treatment guideline for community-acquired pneumonia. Am J Med. 2001;110:451-7.

13. Dean NC, Suchyta MR, Bateman KA, Aronsky D, Hadlock CJ. Implementation of admission decision support for community-acquired pneumonia: a pilot study. Chest. 2000;117:1368-77.

14. Menéndez R, Torres A, Zalacaín R, Aspa J, Martín-Villasclaras JJ, Borderías $L$, et al. Guidelines for the treatment of community-acquired pneumonia: predictors of adherence and outcome. Am J Respir Crit Care. 2005;172:757-62.

15. Capelastegui A, Espana PP, Quintana JM, Areitio I, Gorordo I, Egurrola $M$, et al. Validation of a predictive rule for the management of communityacquired pneumonia. Eur Respir J. 2006;27:151-7. 